\title{
Chromatography Paper: A Novel Solid Support
}
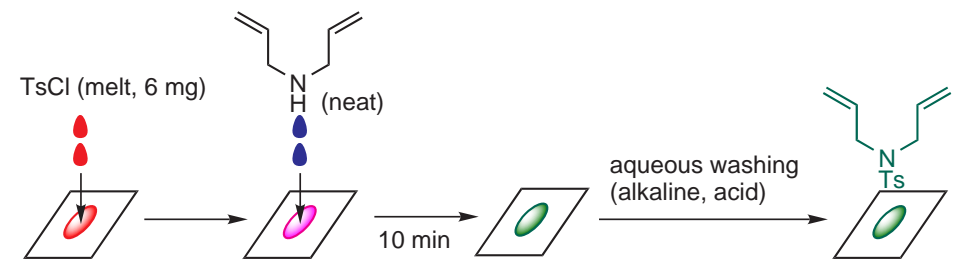

paper $\left(1 \mathrm{~cm}^{2}\right)$

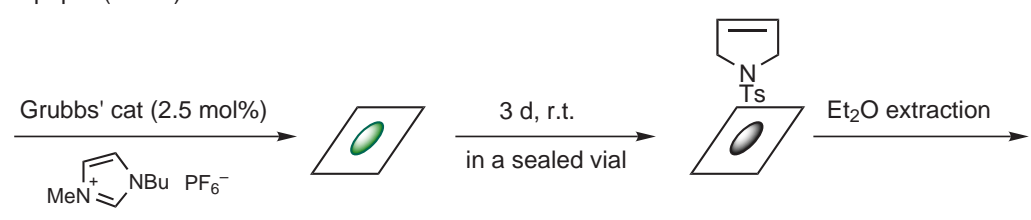

$29 \%$ isolated yield

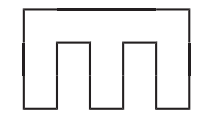

comb-shape reactor

Significance: A cellulose-based chromatography paper was used as a solid support for organic transformations as well as extractive purification. N-tosylation, Grubbs' ring-closing metathesis, and Wadsworth-Emmons olefination were carried out on the chromatography paper support where the substrates and reagents were non-covalently immobilized. For example, as can be seen in the scheme, tosyl chloride was charged on the paper tile as a melt and diallylamine (neat) was added subsequently on to the spot of TsCl. After 10 minutes, the paper reactor was washed with aqueous $\mathrm{NaHCO}_{3}$, water, diluted acid, and water to remove unreacted $\mathrm{TsCl}$ and amine. Ionic liquid solution of Grubbs' catalyst was then loaded onto the reaction spot, and the paper reactor was left for three days in a sealed vial. The paper was extracted with ether to afford a pyrrole product in $29 \%$ isolated yield (single extraction) with high purity (NMR analysis).
Comment: This technology would make the preparation of an encoded library easy by pencil-writing encoding. Parallel reactions of various substrate-reagent combinations can be conveniently carried out on a comb-shaped paper, where each comb-tooth supports a unique substrate/reagent and all comb-teeth (the whole comb) can be washed at once. 